\title{
Interferência de Plantas Concorrentes em ArRoz Irrigado MODIFICADA POR MÉTOdos CULTURAIS ${ }^{1}$
}

\author{
Interference of Concurrent Plants in Flooded Rice Modified by Cultural Methods
}

FLECK, N.G. ${ }^{2}$, AGOSTINETTO, D. ${ }^{3}$, RIZZARDI, M.A. ${ }^{3}$, BIANCHI, M.A. ${ }^{3}$ e MENEZES, V.G. ${ }^{4}$

\begin{abstract}
RESUMO - A otimização de um programa de controle de plantas daninhas depende da previsão precoce e confiável do impacto destas sobre o rendimento da cultura. Este trabalho teve por objetivos avaliar os efeitos de cultivares e de arranjos de plantas na perda de rendimento de grãos em arroz irrigado e identificar a variável explicativa que proporcione melhor ajuste ao modelo testado. Foram conduzidos dois experimentos em campo, na estação estival de crescimento 2000/01. Os tratamentos constaram de três cultivares de arroz (BRS-38 Ligeirinho, IRGA 417 e BR-IRGA 409), dois espaçamentos entre linhas (15 e $25 \mathrm{~cm}$ ) e populações (dez e seis níveis para o primeiro e segundo experimentos, respectivamente) do cultivar de arroz EEA 406, simulando infestação de arroz-vermelho. Os dados foram analisados utilizando-se o modelo de regressão não-linear da hipérbole retangular, ajustado de modo independente para os três fatores estudados. Os resultados do primeiro experimento foram utilizados para avaliar o ajuste dos dados ao modelo e os do segundo para validar o modelo obtido no primeiro experimento. O cultivar IRGA 417 apresentou maior habilidade competitiva com o cultivar simulador de arroz-vermelho do que os outros dois. A redução no espaçamento entre linhas aumentou a habilidade competitiva dos cultivares de arroz em relação ao cultivar concorrente. O modelo da hipérbole retangular foi apropriado para estimar perdas de rendimento de grãos de arroz irrigado por interferência de plantas concorrentes. A variável explicativa área foliar do cultivar EEA 406, em geral, apresentou equivalência à população de plantas como variável independente no modelo matemático testado.
\end{abstract}

Palavras-chave: Oryza sativa, arroz-vermelho, competição, modelagem.

\begin{abstract}
The optimization of a weed control program depends on early and reliable prediction of the impact of weeds on crop yield. This research aimed to evaluate the effects of rice cultivars and plant arrangements on flooded rice grain yield loss and to identify the explicative variable that provides the best adjustment to the model tested. Two field experiments were carried out during the 2000/2001 growing season. Treatments consisted of three rice cultivars (BRS-38 Ligeirinho, IRGA 417, and BR-IRGA 409), two row widths (15 and $25 \mathrm{~cm}$ ), and plant populations (ten and six levels for the first and second experiments, respectively) of the rice cultivar EEA 406, mimicking red rice infestation.. Data were analyzed using the non - linear regression model of rectangular hyperbole, adjusted independently for the three studied factors. Results of the first experiment were used to evaluate data adjustment to the model and results of the second to validate the model generated in the first. IRGA 417 cultivar presented greater competitive ability than the other two. Reduction of row width increased the ability of rice cultivars to compete with the concurrent genotype. The rectangular hyperbolic model was suitable to estimate flooded rice grain yield loss due to interference of concurrent plants. The explicative variable leaf area of EEA 406 cultivar, in general, was equivalent to plant population as an independent variable in the mathematical model tested.
\end{abstract}

Key words: Oryza sativa, red rice, competition, modeling.

Recebido para publicação em 21.10.2003 e na forma revisada em 5.3.2004.

Eng.-Agr., Ph.D., Prof. da Faculdade de Agronomia da Universidade Federal do Rio Grande do Sul - UFRGS, Caixa Postal 15100, 90001-970 Porto Alegre-RS; ${ }^{3}$ Eng.-Agr., M.S., Aluno do Programa de Pós-Graduação em Fitotecnia da UFRGS, <agostinetto@agicultura.gov.br>. ${ }^{4}$ Eng.-Agr., M.S., Pesquisador do Instituto Rio-Grandense do Arroz, Caixa Postal 29, $94930-$ 030 Cachoeirinha-RS. 


\section{INTRODUÇÃO}

A utilização de métodos culturais para manejo de plantas daninhas é especialmente importante em situações em que o controle químico para determinada espécie seja limitado pela indisponibilidade de herbicidas seletivos (Blackshaw et al., 2000). No caso do arroz-vermelho (Oryza sativa), várias práticas de manejo podem minimizar sua interferência em arroz cultivado; dentre elas, destacam-se o uso de cultivares competitivos e a mudança no arranjo de plantas.

Trabalhos de pesquisa demonstram existir variabilidade na habilidade competitiva de cultivares de arroz com plantas daninhas (Ni et al., 2000; Balbinot Jr. et al., 2003). O incremento da capacidade competitiva de certos cultivares tem sido atribuído aos seguintes fatores: emergência precoce, maior vigor das plântulas, aumento da taxa de expansão foliar, rápido desenvolvimento do dossel, maior estatura de planta, ciclo precoce e aumento no tamanho de raízes (Berkowitz, 1988). Desse modo, a utilização de cultivares com maior habilidade competitiva pode, em parte, reduzir a intensidade de competição das plantas daninhas.

Quando duas espécies com características semelhantes estão competindo no mesmo nicho, a eqüidistância entre os indivíduos assume grande importância, ao aumentar a habilidade competitiva de uma delas. Além disso, o arranjo e a população de plantas adequados são essenciais para obtenção de elevados rendimento e qualidade de grãos (Gravois \& Helms, 1992). Diversos trabalhos de pesquisa foram realizados com o propósito de definir o espaçamento entre linhas e a população de plantas de arroz que otimizem o rendimento de grãos da cultura (Menezes \& Silva, 1998; Rieffel Neto et al., 2000). No entanto, poucos deles avaliaram a variação da habilidade competitiva de cultivares de arroz, pelo arranjo de plantas, quando em competição com arrozvermelho. Entre as vantagens da redução do espaçamento entre linhas podem-se citar: crescimento e fechamento mais rápido do dossel, com melhor uso da radiação solar, diminuição dos efeitos de competição intra-específica e aumento da competitividade com plantas daninhas.
Melhorar a predição de perda de rendimento dos cultivos é questão essencial quando se trata de desenvolver estratégias alternativas de manejo de plantas daninhas. Isso é possível obter pela utilização de modelos de competição entre culturas e plantas daninhas, os quais podem simular a complexa e dinâmica interação entre espécies. A relação entre perda de rendimento da cultura e variáveis explicativas, como população, massa seca, cobertura do solo e área foliar das plantas daninhas, pode ser descrita adequadamente pela equação nãolinear da hipérbole retangular (Cousens, 1985). Segundo esse autor, o modelo da hipérbole apresenta dois parâmetros com significado biológico e agronômico ( $i$ e $a$ ), os quais podem ser usados juntos, como índices de competitividade.

A hipótese do trabalho foi de que a redução no espaçamento entre linhas e a utilização de cultivares de ciclo curto reduzem o grau de interferência negativa do cultivar EEA 406, simulador de arroz-vermelho, no arroz cultivado. O objetivo foi avaliar os efeitos de cultivares e do arranjo de plantas na perda de rendimento de grãos de arroz irrigado, bem como identificar a variável explicativa que forneça melhor ajuste ao modelo matemático da hipérbole retangular.

\section{MATERIAL E MÉTODOS}

Foram conduzidos dois experimentos em campo, na Estação Experimental do Arroz, pertencente ao Instituto Rio-Grandense do Arroz (IRGA), durante a estação de crescimento 2000/01. O delineamento experimental utilizado em ambos os experimentos foi completamente casualizado, com uma unidade experimental por combinação de fatores. Cada unidade experimental (parcela) foi composta por área de $9 \mathrm{~m}^{2}$ ou $6,75 \mathrm{~m}^{2}$ para o primeiro e segundo experimentos, respectivamente. Os tratamentos, dispostos em esquema fatorial, constaram de três cultivares de arroz (BRS-38 Ligeirinho, ciclo muito curto; IRGA 417, ciclo curto; e BR-IRGA 409, ciclo médio), de dois espaçamentos entre linhas ( 15 e $25 \mathrm{~cm}$ ) e de populações do cultivar EEA 406, o qual, por apresentar características morfológicas semelhantes às do arroz-vermelho, exerceu a função de simulador do arroz daninho. A não-utilização do próprio arroz-vermelho como infestante 
natural no segundo experimento deve-se ao nível geralmente elevado de dormência de suas sementes (Agostinetto et al., 2001), o que leva à emergência desuniforme, dificultando o estabelecimento de populações predeterminadas no tempo e no espaço. No primeiro experimento foram utilizados dez níveis do cultivar simulador, obtendo-se populações máximas de 264 e 278, 164 e 270 e 152 e 192 plantas m $^{-2}$ para os cultivares BRS-38 Ligeirinho, IRGA 417 e BR-IRGA 409, nos espaçamentos entre linhas de 15 e $25 \mathrm{~cm}$, respectivamente. No segundo experimento, cujo objetivo foi validar o modelo ajustado para o primeiro experimento, foram utilizados seis níveis do cultivar competidor, alcançando-se populações máximas de 48 e 74 , 62 e 64 e 136 e 182 plantas $\mathrm{m}^{-2}$, respectivamente, para os cultivares e espaçamentos mencionados acima.

O preparo do solo foi realizado segundo o sistema convencional. Como adubação, utilizaram-se na semeadura $10 \mathrm{~kg}$ de $\mathrm{N} \mathrm{ha}^{-1}, 20 \mathrm{~kg}$ de $\mathrm{P}_{2} \mathrm{O}_{5}$ ha $^{-1}$ e $40 \mathrm{~kg}$ de $\mathrm{K}_{2} \mathrm{O} \mathrm{ha}^{-1} \mathrm{e}$, em cobertura, $80 \mathrm{~kg}$ de $\mathrm{N} \mathrm{ha}^{-1}$, fracionados em três épocas de aplicação. A semeadura do arroz foi realizada em 17/11/2000 e a emergência ocorreu sete dias após. A adubação, o controle de plantas daninhas e as demais práticas de manejo utilizadas foram aqueles preconizados pela pesquisa (Embrapa, 1999).

Em ambos os experimentos, aos $14 \mathrm{e}$ 28 dias após a emergência (DAE), realizaram-se avaliações das variáveis explicativas população de plantas, cobertura do solo pela folhagem, massa seca da parte aérea e área foliar para o cultivar simulador. A determinação da população de plantas foi feita mediante a contagem dos indivíduos presentes em duas áreas de $0,25 \mathrm{~m}^{2}(0,5 \times 0,5 \mathrm{~m}) \mathrm{em}$ cada parcela. A cobertura do solo foi avaliada visualmente, de modo individual, por dois avaliadores, utilizando-se escala percentual, em que a nota zero correspondeu à ausência de cobertura do solo e a nota 100 representou cobertura completa do solo. A massa seca do simulador foi avaliada pela coleta e secagem em estufa, até peso constante, das plantas presentes em área de $0,25 \mathrm{~m}^{2}(0,5 \mathrm{x}$ $0,5 \mathrm{~m}$ ) por parcela. A área foliar foi determinada com um integrador eletrônico de área foliar, nas plantas colhidas para avaliação de massa seca.
O rendimento de grãos foi quantificado pela colheita do arroz nas áreas centrais de cada parcela. Essa colheita foi realizada em épocas distintas, de acordo com a maturação de cada cultivar, quando o teor de umidade dos grãos estava em torno de $22 \%$. Após pesagem dos grãos, foi determinada sua umidade, sendo os pesos corrigidos para teor de $13 \%$ de umidade.

Para avaliar a existência ou não de interação dos fatores estudados, realizou-se análise de variância para dados de perdas percentuais de rendimento de grãos, considerando-se o nível de 5\% de probabilidade para significância de efeitos individuais dos fatores e de $15 \%$ para efeitos de interação. Assim, após ser constatada significância estatística, as relações entre perdas percentuais de rendimento de grãos do arroz cultivado e as variáveis explicativas foram determinadas separadamente para cada cultivar e espaçamento entre linhas, ajustando-se os dados ao modelo de regressão nãolinear da hipérbole retangular, proposto por Cousens em 1985:

$$
\operatorname{Pr}=\frac{(i * X)}{\left[1+\left(\frac{i}{a}\right) * X\right]}
$$

em que: $\operatorname{Pr}=$ perda de rendimento (\%); $X=$ população, cobertura do solo, massa seca da parte aérea ou área foliar do cultivar simulador de arroz-vermelho; $i=$ perda de rendimento (\%) por unidade do genótipo competidor quando a respectiva variável se aproxima de zero; e $a=$ perda de rendimento (\%) quando a variável em questão tende ao infinito.

Os valores de cobertura do solo (\%), massa seca da parte aérea $\left(\mathrm{g} \mathrm{m}^{-2}\right)$ e área foliar $\left(\mathrm{m}^{2}\right)$ foram multiplicados por 100, evitando-se assim a necessidade de correção desses valores no modelo. O ajuste dos dados ao modelo foi feito através do procedimento Proc Nlin do programa computacional SAS (SAS, 1989). No procedimento de cálculos utilizou-se o método de Gauss-Newton, o qual, por sucessivas iterações, estima os valores dos parâmetros nos quais a soma dos quadrados dos desvios das observações em relação aos valores ajustados seja mínima (Ratkowsky, 1983). Nos casos em que a estimativa para o parâmetro $a$ (perda máxima de rendimento) foi superestimada, o valor da assíntota foi limitado em 100\%. Esse 
procedimento é recomendado quando se trata de evitar a obtenção de perdas de rendimento superiores a $100 \%$, as quais são biologicamente irreais (Yenish et al., 1997; Askew \& Wilcut, 2001). O valor da estatística $F$, em nível de $5 \%$ de probabilidade, foi utilizado como critério de ajuste dos dados ao modelo. O critério de aceitação do melhor ajuste dos dados ao modelo baseou-se no coeficiente de determinação $\left(R^{2}\right)$ e na soma de quadrados do resíduo (SQR), de modo que maior valor do primeiro e menor valor do segundo representavam ajuste mais satisfatório.

A validação do modelo ajustado foi feita mediante análise de correlação linear simples entre as perdas de rendimento observadas no segundo experimento e as estimadas com base nos parâmetros ajustados no primeiro experimento.

\section{RESULTADOS E DISCUSSÃo}

O cultivar BRS-38 Ligeirinho apresentou valores da estatística $F$ significativos para todas as variáveis explicativas e todos os espaçamentos entre linhas testados (Tabela 1). De modo geral, os dados ajustaram-se adequadamente ao modelo da hipérbole retangular, à exceção da variável cobertura do solo no espaçamento de $15 \mathrm{~cm}$ na primeira época de avaliação, a qual apresentou baixo valor de $\mathrm{R}^{2} \mathrm{e}$ elevada SQR, o que caracteriza um baixo ajuste ao modelo. Os valores de $\mathrm{R}^{2}$ estimados para o espaçamento entre linhas de $25 \mathrm{~cm}$ foram

Tabela 1 - Ajustes obtidos para perda do rendimento de grãos do cultivar de arroz BRS-38 Ligeirinho em função do espaçamento entre linhas e em resposta a variáveis avaliadas no cultivar EEA 406, simulador de arroz-vermelho. IRGA/Cachoeirinha-RS, 2000/01

\begin{tabular}{|c|c|c|c|c|c|}
\hline \multirow[t]{2}{*}{ Variável explicativa } & \multicolumn{2}{|c|}{ Parâmetro ${ }^{1 /}$} & \multirow{2}{*}{$\begin{array}{l}\text { Coeficiente de } \\
\text { determinação } \\
\left(\mathrm{R}^{2}\right)\end{array}$} & \multirow{2}{*}{$\begin{array}{l}\text { Soma de quadrados } \\
\text { do resíduo }\end{array}$} & \multirow{2}{*}{$\begin{array}{c}\text { Estatística } \\
\text { F }\end{array}$} \\
\hline & $\bar{i}$ & $a$ & & & \\
\hline \multicolumn{6}{|c|}{$14 \mathrm{DAE}^{2 /}$} \\
\hline $\begin{array}{l}\text { População de plantas } \\
\qquad 15 \mathrm{~cm}^{3 /} \\
25 \mathrm{~cm}\end{array}$ & $\begin{array}{l}0,63 \\
0,70\end{array}$ & $\begin{array}{l}100,0 \\
100,0\end{array}$ & $\begin{array}{l}0,64 \\
0,75\end{array}$ & $\begin{array}{l}1266,0 \\
1921,3\end{array}$ & $\begin{array}{l}36,5^{*} \\
38,4^{*}\end{array}$ \\
\hline $\begin{array}{c}\text { Cobertura do solo } \\
15 \mathrm{~cm} \\
25 \mathrm{~cm}\end{array}$ & $\begin{array}{l}0,04 \\
0,06\end{array}$ & $\begin{array}{l}100,0 \\
100,0\end{array}$ & $\begin{array}{l}0,37 \\
0,68\end{array}$ & $\begin{array}{l}2224,3 \\
2390,6\end{array}$ & $\begin{array}{l}16,9 * \\
29,1 *\end{array}$ \\
\hline $\begin{array}{c}\text { Massa seca } \\
15 \mathrm{~cm} \\
25 \mathrm{~cm}\end{array}$ & $\begin{array}{l}0,12 \\
0,14\end{array}$ & $\begin{array}{l}100,0 \\
100,0\end{array}$ & $\begin{array}{l}0,67 \\
0,72\end{array}$ & $\begin{array}{l}1179,0 \\
2079,5\end{array}$ & $\begin{array}{l}39,8^{*} \\
34,8^{*}\end{array}$ \\
\hline $\begin{array}{c}\text { Ârea foliar } \\
15 \mathrm{~cm} \\
25 \mathrm{~cm}\end{array}$ & $\begin{array}{l}0,001 \\
0,001\end{array}$ & $\begin{array}{l}100,0 \\
100,0\end{array}$ & $\begin{array}{l}0,66 \\
0,77\end{array}$ & $\begin{array}{l}1191,8 \\
1741,0\end{array}$ & $\begin{array}{l}39,3^{*} \\
43,3^{*}\end{array}$ \\
\hline \multicolumn{6}{|c|}{28 DAE } \\
\hline $\begin{array}{l}\text { População de plantas } \\
15 \mathrm{~cm} \\
25 \mathrm{~cm}\end{array}$ & $\begin{array}{l}0,75 \\
0,72\end{array}$ & $\begin{array}{l}100,0 \\
100,0\end{array}$ & $\begin{array}{l}0,67 \\
0,67\end{array}$ & $\begin{array}{l}1178,3 \\
2509,1\end{array}$ & $\begin{array}{l}39,9 * \\
27,3 *\end{array}$ \\
\hline $\begin{array}{l}\text { Cobertura do solo } \\
15 \mathrm{~cm} \\
25 \mathrm{~cm}\end{array}$ & $\begin{array}{l}0,05 \\
0,06\end{array}$ & $\begin{array}{l}100,0 \\
100,0\end{array}$ & $\begin{array}{l}0,56 \\
0,69\end{array}$ & $\begin{array}{l}1538,5 \\
2345,6\end{array}$ & $\begin{array}{l}28,4 * \\
29,8 *\end{array}$ \\
\hline $\begin{array}{c}\text { Massa seca } \\
15 \mathrm{~cm} \\
25 \mathrm{~cm}\end{array}$ & $\begin{array}{l}0,02 \\
0,03\end{array}$ & $\begin{array}{l}100,0 \\
100,0\end{array}$ & $\begin{array}{l}0,54 \\
0,68\end{array}$ & $\begin{array}{l}1620,1 \\
2386,9 .\end{array}$ & $\begin{array}{l}26,5 * \\
29,1 *\end{array}$ \\
\hline $\begin{array}{l}\text { Arrea foliar } \\
15 \mathrm{~cm} \\
25 \mathrm{~cm}\end{array}$ & $\begin{array}{l}0,0002 \\
0,0002\end{array}$ & $\begin{array}{l}100,0 \\
100,0\end{array}$ & $\begin{array}{l}0,62 \\
0,69\end{array}$ & $\begin{array}{l}1351,3 \\
2335,9\end{array}$ & $\begin{array}{l}33,6^{*} \\
30,0^{*}\end{array}$ \\
\hline
\end{tabular}

$\stackrel{1}{ } i$ e $a=$ perdas de rendimento $(\%)$ por unidade do cultivar simulador quando o valor da variável se aproxima de zero ou tende ao infinito, respectivamente; ${ }^{2 /}$ Dias após a emergência do arroz cultivado; ${ }^{3 /}$ Espaçamento entre linhas do arroz cultivado; e $*$ significativo a $5 \%$ de probabilidade. 
superiores aos obtidos para o espaçamento de $15 \mathrm{~cm}$, à exceção da variável população de plantas na segunda avaliação. Isso pode ser atribuído ao fato de o maior espaçamento entre linhas facilitar a identificação das plantas do cultivar concorrente, o que pode ter auxiliado na avaliação mais precisa das variáveis explicativas.

Os valores estimados para o parâmetro $i$ tenderam a aumentar com o incremento do espaçamento entre linhas, à exceção das variáveis população de plantas na segunda avaliação e área foliar em ambas as avaliações (Tabela 1). Esses resultados podem estar associados ao ciclo do cultivar e às características morfofisiológicas deste. Cultivares de ciclo muito curto apresentam menor fase vegetativa, havendo, conseqüentemente, menor período para acúmulo de fotoassimilados para investir em estrutura da planta. O cultivar BRS-38 Ligeirinho caracteriza-se por possuir ciclo muito curto (até 100 dias) e média a alta capacidade de afilhamento (Arroz irrigado: Recomendações, 1997). Assim, o fechamento das entrelinhas pelo dossel só ocorre em estádio mais avançado de desenvolvimento, comparativamente aos outros cultivares.

Os valores para o parâmetro $a$ foram superestimados pelo modelo; desse modo, restringiu-se a assintota a 100\% de perda, para todas as variáveis testadas (Tabela 1). Ao analisar comparativamente as variáveis explicativas, e considerando as duas épocas de avaliação, em geral, as variáveis que melhor se ajustaram ao modelo foram população de plantas e área foliar.

Para o cultivar IRGA 417, os dados ajustaram-se satisfatoriamente ao modelo (Tabela 2); para todas as variáveis e espaçamentos entre linhas estudados, os valores da estatística $\mathrm{F}$ foram significativos. Quando o espaçamento entre linhas foi maior, em geral, os valores de $\mathrm{R}^{2}$ aumentaram e os de SQR diminuíram, o que caracteriza melhor ajuste ao modelo, neste caso. Para espaçamento entre linhas de $15 \mathrm{~cm}$, os valores de $\mathrm{R}^{2}$ tenderam a ser maiores para as variáveis população de plantas e área foliar; já para o de $25 \mathrm{~cm}$, as variáveis que tenderam a se ajustar melhor ao modelo foram área foliar e massa seca.

Para o parâmetro $i$, os valores estimados tenderam a duplicar de valor com o aumento no espaçamento entre linhas, na primeira época de avaliação (Tabela 2). Por ser o parâmetro $i$ um índice usado para comparar a competitividade relativa entre espécies (Swinton et al., 1994), constata-se que a redução no espaçamento entre linhas praticamente dobrou a competitividade do cultivar de arroz IRGA 417 com a competidora, para as variáveis testadas na primeira avaliação.

No menor espaçamento entre linhas, os valores estimados para a assintota superaram 100\% (Tabela 2). De acordo com Ngouajio et al. (1999), os modelos hiperbólicos usados para estimar perdas de rendimento apresentam resposta assintótica para altos níveis de infestação de plantas daninhas. Esse comportamento pode resultar em superestimação do impacto de plantas daninhas e redução do nível de perda de rendimento para tomada de decisão de controle (Swinton \& Lyford, 1996).

Novamente, as variáveis explicativas população de plantas e área foliar do cultivar simulador de arroz-vermelho foram as que apresentaram melhores ajustes dos dados ao modelo (Tabela 2).

Os ajustes dos dados ao modelo da hipérbole retangular, para o cultivar BR-IRGA 409, foram significativos para todas as variáveis explicativas, incluindo ambos os espaçamentos entre linhas e as épocas de avaliação (Tabela 3). Os valores de $\mathrm{R}^{2}$ variaram de 0,76 a 0,92 , sendo satisfatórios em termos de ajuste.

Os valores obtidos para o parâmetro $i$ diminuíram à medida que o espaçamento entre linhas diminuiu, com exceção da variável área foliar na segunda avaliação, condição em que os valores foram idênticos. As médias das duas épocas de avaliação demonstraram que o aumento do espaçamento entre linhas elevou os valores do parâmetro $i$ em 98, 184, 142 e 34\%, respectivamente, para as variáveis população de plantas, cobertura do solo, massa seca da parte aérea e área foliar (Tabela 3). De modo semelhante ao observado para o cultivar IRGA 417, o aumento do espaçamento entre linhas reduziu a competitividade do cultivar BR-IRGA 409 com o cultivar simulador, situação ocorrida em todas as variáveis explicativas e épocas de avaliação, exceto área foliar na segunda avaliação. Já para o parâmetro $a$, houve novamente superestimação do valor da assíntota, especialmente no maior espaçamento testado. 
Tabela 2 - Ajustes obtidos para perda do rendimento de grãos do cultivar de arroz IRGA 417 em função do espaçamento entre linhas e em resposta a variáveis avaliadas no cultivar EEA 406, simulador de arroz-vermelho. IRGA/Cachoeirinha-RS, 2000/01

\begin{tabular}{|c|c|c|c|c|c|}
\hline \multirow[t]{2}{*}{ Variável explicativa } & \multicolumn{2}{|c|}{ Parâmetro ${ }^{1 /}$} & \multirow{2}{*}{$\begin{array}{l}\text { Coeficiente de } \\
\text { determinação } \\
\left(\mathrm{R}^{2}\right)\end{array}$} & \multirow{2}{*}{$\begin{array}{l}\text { Soma de quadrados } \\
\text { do resíduo }\end{array}$} & \multirow{2}{*}{$\begin{array}{c}\text { Estatística } \\
\text { F }\end{array}$} \\
\hline & $\bar{i}$ & $a$ & & & \\
\hline \multicolumn{6}{|c|}{$14 \mathrm{DAE}^{2 /}$} \\
\hline $\begin{array}{l}\text { População de plantas } \\
15 \mathrm{~cm}^{3 /} \\
25 \mathrm{~cm}\end{array}$ & $\begin{array}{l}0,25 \\
0,51\end{array}$ & $\begin{array}{r}100,0 \\
91,6\end{array}$ & $\begin{array}{l}0,68 \\
0,85\end{array}$ & $\begin{array}{l}538,6 \\
540,5\end{array}$ & $\begin{array}{l}17,7^{*} \\
29,7^{*}\end{array}$ \\
\hline $\begin{array}{c}\text { Cobertura do solo } \\
15 \mathrm{~cm} \\
25 \mathrm{~cm}\end{array}$ & $\begin{array}{l}0,02 \\
0,04\end{array}$ & $\begin{array}{l}100,0 \\
100,0\end{array}$ & $\begin{array}{l}0,65 \\
0,76\end{array}$ & $\begin{array}{l}585,9 \\
876,2\end{array}$ & $\begin{array}{l}15,6^{*} \\
43,6^{*}\end{array}$ \\
\hline $\begin{array}{l}\text { Massa seca } \\
15 \mathrm{~cm} \\
25 \mathrm{~cm} \\
\text { Área foliar } \\
15 \mathrm{~cm} \\
25 \mathrm{~cm}\end{array}$ & $\begin{array}{l}0,06 \\
0,12 \\
0,0005 \\
0,001\end{array}$ & $\begin{array}{r}100,0 \\
83,9 \\
100,0 \\
73,3\end{array}$ & $\begin{array}{l}0,47 \\
0,86 \\
0,63 \\
0,88\end{array}$ & $\begin{array}{l}887,5 \\
518,0 \\
623,9 \\
436,1\end{array}$ & $\begin{array}{r}7,6^{*} \\
35,6^{*} \\
14,2^{*} \\
43,0^{*}\end{array}$ \\
\hline \multicolumn{6}{|c|}{28 DAE } \\
\hline $\begin{array}{l}\text { População de plantas } \\
15 \mathrm{~cm} \\
25 \mathrm{~cm}\end{array}$ & $\begin{array}{l}0,49 \\
0,82\end{array}$ & $\begin{array}{r}100,0 \\
77,3 \\
\end{array}$ & $\begin{array}{l}0,70 \\
0,87\end{array}$ & $\begin{array}{l}512,4 \\
466,5\end{array}$ & $\begin{array}{l}14,0 * \\
34,9 *\end{array}$ \\
\hline $\begin{array}{c}\text { Cobertura do solo } \\
15 \mathrm{~cm} \\
25 \mathrm{~cm}\end{array}$ & $\begin{array}{l}0,03 \\
0,05\end{array}$ & $\begin{array}{r}100,0 \\
100,0\end{array}$ & $\begin{array}{l}0,64 \\
0,87\end{array}$ & $\begin{array}{l}612,4 \\
476,1\end{array}$ & $\begin{array}{l}14,6 * \\
87,9 *\end{array}$ \\
\hline $\begin{array}{l}\text { Massa seca } \\
15 \mathrm{~cm} \\
25 \mathrm{~cm}\end{array}$ & $\begin{array}{l}0,02 \\
0,03\end{array}$ & $\begin{array}{r}100,0 \\
80,7 \\
\end{array}$ & $\begin{array}{l}0,67 \\
0,88\end{array}$ & $\begin{array}{l}560,5 \\
434,0\end{array}$ & $\begin{array}{l}16,7 * \\
43,2 *\end{array}$ \\
\hline $\begin{array}{l}\text { Área foliar } \\
15 \mathrm{~cm} \\
25 \mathrm{~cm}\end{array}$ & $\begin{array}{l}0,0002 \\
0,0002\end{array}$ & $\begin{array}{r}100,0 \\
69,6\end{array}$ & $\begin{array}{l}0,70 \\
0,87\end{array}$ & $\begin{array}{l}504,7 \\
464,4\end{array}$ & $\begin{array}{l}19,4^{*} \\
40,2 *\end{array}$ \\
\hline
\end{tabular}

${ }^{1 /} i$ e $a=$ perdas de rendimento (\%) por unidade do cultivar simulador quando o valor da variável se aproxima de zero ou tende ao infinito, respectivamente; ${ }^{2 /}$ Dias após a emergência do arroz cultivado; ${ }^{3 /}$ Espaçamento entre linhas do arroz cultivado; e $*$ significativo a $5 \%$ de probabilidade.

Os ajustes obtidos para as variáveis explicativas ao modelo, nas avaliações relativas ao espaçamento entre linhas de $15 \mathrm{~cm}$, foram praticamente equivalentes (Tabela 3 ). No entanto, para espaçamento de $25 \mathrm{~cm}$, a variável cobertura do solo ajustou-se melhor ao modelo do que as demais variáveis. Isso pode decorrer da maior facilidade na estimativa visual de cobertura vegetal do solo pelo cultivar competidor, em razão do maior espaçamento entre linhas. Levando em conta os valores de $\mathrm{R}^{2} \mathrm{e}$ SQR, verifica-se que, na média das duas épocas de avaliação, as variáveis cobertura do solo e população de plantas foram as que apresentaram maior ajuste ao modelo; massa seca e área foliar foram equivalentes.
Ao comparar conjuntamente os três cultivares de arroz, verifica-se que os valores estimados para o parâmetro $i$ foram relativamente baixos para todas as variáveis explicativas (Tabelas 1, 2 e 3). Tomando-se como exemplo a variável população de plantas, a análise comparativa dos três cultivares na primeira avaliação, com base no parâmetro $i$, demonstra que o cultivar IRGA 417 apresentou maior habilidade competitiva com o cultivar concorrente, em ambos os espaçamentos testados. BRS-38 Ligeirinho mostrou menor potencial competitivo no espaçamento de $15 \mathrm{~cm}$. Contudo, na segunda avaliação, os cultivares IRGA 417 e BR-IRGA 409 mostraram resposta semelhante. 
Tabela 3 - Ajustes obtidos para perda do rendimento de grãos do cultivar de arroz BR-IRGA 409 em função do espaçamento entre linhas e em resposta a variáveis avaliadas no cultivar EEA 406, simulador de arroz-vermelho. IRGA/CachoeirinhaRS, 2000/01

\begin{tabular}{|c|c|c|c|c|c|}
\hline \multirow{2}{*}{ Variáveis explicativas } & \multicolumn{2}{|c|}{ Parâmetros ${ }^{1 /}$} & \multirow{2}{*}{$\begin{array}{l}\text { Coeficiente de } \\
\text { determinação } \\
\left(\mathrm{R}^{2}\right)\end{array}$} & \multirow{2}{*}{$\begin{array}{l}\text { Soma de quadrados } \\
\text { do resíduo }\end{array}$} & \multirow{2}{*}{$\begin{array}{c}\text { Estatística } \\
\mathrm{F}\end{array}$} \\
\hline & $\bar{i}$ & $\bar{a}$ & & & \\
\hline \multicolumn{6}{|c|}{$14 \mathrm{DAE}^{2 /}$} \\
\hline $\begin{array}{l}\text { População de plantas } \\
15 \mathrm{~cm}^{3 /} \\
25 \mathrm{~cm}\end{array}$ & $\begin{array}{l}0,32 \\
0,77 \ldots\end{array}$ & $\begin{array}{r}81,9 \\
100,0\end{array}$ & $\begin{array}{l}0,85 \\
0,87\end{array}$ & $\begin{array}{l}318,2 \\
563,6 .\end{array}$ & $\begin{array}{l}25,0 * \\
92,8 *\end{array}$ \\
\hline $\begin{array}{c}\text { Cobertura do solo } \\
15 \mathrm{~cm} \\
25 \mathrm{~cm}\end{array}$ & $\begin{array}{l}0,02 \\
0,06\end{array}$ & $\begin{array}{l}100,0 \\
100,0\end{array}$ & $\begin{array}{l}0,81 \\
0,91\end{array}$ & $\begin{array}{l}398,1 \\
391,2\end{array}$ & $\begin{array}{r}49,6^{*} \\
144,6^{*}\end{array}$ \\
\hline $\begin{array}{c}\text { Massa seca } \\
15 \mathrm{~cm} \\
25 \mathrm{~cm}\end{array}$ & $\begin{array}{l}0,06 \\
0,20\end{array}$ & $\begin{array}{l}100,0 \\
100,0\end{array}$ & $\begin{array}{l}0,85 \\
0,84\end{array}$ & $\begin{array}{l}311,4 \\
691,9\end{array}$ & $\begin{array}{l}65,8^{*} \\
77,8^{*}\end{array}$ \\
\hline $\begin{array}{c}\text { Ârea foliar } \\
15 \mathrm{~cm} \\
25 \mathrm{~cm} \\
\end{array}$ & $\begin{array}{l}0,0006 \\
0,001 \\
\end{array}$ & $\begin{array}{r}70,7 \\
100,0 \\
\end{array}$ & $\begin{array}{l}0,85 \\
0,81 \\
\end{array}$ & $\begin{array}{l}324,6 \\
829,4 \\
\end{array}$ & $\begin{array}{l}27,9 * \\
60,1^{*}\end{array}$ \\
\hline \multicolumn{6}{|c|}{28 DAE } \\
\hline $\begin{array}{l}\text { População de plantas } \\
15 \mathrm{~cm} \\
25 \mathrm{~cm}\end{array}$ & $\begin{array}{l}0,50 \\
0,77\end{array}$ & $\begin{array}{l}100,0 \\
100,0\end{array}$ & $\begin{array}{l}0,82 \\
0,91\end{array}$ & $\begin{array}{l}395,6 \\
364,3\end{array}$ & $\begin{array}{r}49,9 * \\
155,9 *\end{array}$ \\
\hline $\begin{array}{c}\text { Cobertura do solo } \\
15 \mathrm{~cm} \\
25 \mathrm{~cm}\end{array}$ & $\begin{array}{l}0,03 \\
0,08\end{array}$ & $\begin{array}{r}89,7 \\
100,0\end{array}$ & $\begin{array}{l}0,90 \\
0,92\end{array}$ & $\begin{array}{l}224,4 \\
322,3\end{array}$ & $\begin{array}{r}42,1^{*} \\
177,4^{*}\end{array}$ \\
\hline 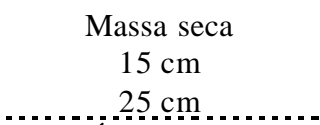 & $\begin{array}{l}0,02 \\
0,03 \ldots\end{array}$ & $\begin{array}{r}100,0 \\
100,0\end{array}$ & $\begin{array}{l}0,81 \\
0,76\end{array}$ & $\begin{array}{r}410,1 \\
1025,1\end{array}$ & $\begin{array}{l}47,8^{*} \\
49,6 *\end{array}$ \\
\hline $\begin{array}{c}\text { Área foliar } \\
15 \mathrm{~cm} \\
25 \mathrm{~cm}\end{array}$ & $\begin{array}{l}0,0002 \\
0,0002 \\
\end{array}$ & $\begin{array}{l}100,0 \\
100,0\end{array}$ & $\begin{array}{l}0,86 \\
0,76 \\
\end{array}$ & $\begin{array}{r}299,0 \\
1013,4 \\
\end{array}$ & $\begin{array}{l}69,0 * \\
47,6 *\end{array}$ \\
\hline
\end{tabular}

${ }^{1 /} i$ e $a=$ perdas de rendimento (\%) por unidade do cultivar simulador quando o valor da variável se aproxima de zero ou tende ao infinito, respectivamente; ${ }^{2 /}$ Dias após a emergência do arroz cultivado; ${ }^{3 /}$ Espaçamento entre linhas do arroz cultivado; e $*$ significativo a $5 \%$ de probabilidade.

Para o cultivar IRGA 417, na avaliação de 14 DAE, os valores estimados para $i$ foram aproximadamente o dobro no espaçamento entre linhas de $25 \mathrm{~cm}$, em relação ao de $15 \mathrm{~cm}$ (Tabela 2). Em se tratando do cultivar BR-IRGA 409, exceto para a variável área foliar, os valores estimados para o parâmetro $i$, na avaliação de $14 \mathrm{DAE}$, foram maiores que o dobro no espaçamento de $25 \mathrm{~cm}$, quando comparado aos de $15 \mathrm{~cm}$ (Tabela 3). Isso demonstra que, nessa avaliação, o cultivar BR-IRGA 409 foi mais sensível à competição do simulador do que IRGA 417. No entanto, na avaliação realizada aos $28 \mathrm{DAE}$, ambos os cultivares mostraram maior equilíbrio nas estimativas do parâmetro $i$, em resposta às variações no espaçamento entre linhas, exceto para a variável cobertura do solo, em que BR-IRGA 409 continuou a indicar maior impacto da competição do simulador no espaçamento de $25 \mathrm{~cm}$ do que IRGA 417, em relação ao espaçamento de $15 \mathrm{~cm}$.

Considerando a primeira avaliação (14 DAE), época normalmente recomendada para controle de plantas daninhas em pósemergência, e o menor espaçamento $(15 \mathrm{~cm})$, em geral mais utilizado pelos orizicultores do Estado do Rio Grande do Sul, observa-se que o cultivar BRS-38 Ligeirinho apresentou maiores perdas por unidade do cultivar simulador do que os outros dois (Tabelas 1, 2 e 3). Esses resultados corroboram aqueles discutidos quando da análise individual do cultivar 
e, provavelmente, se devem ao ciclo muito curto e às características morfofisiológicas do cultivar, que o tornam pouco competitivo. De modo semelhante, Menezes \& Silva (1998) observaram que o maior ciclo e a maior estatura de planta do cultivar BR-IRGA 410 (ciclo médio) proporcionaram vantagens em relação ao cultivar IRGA 416 (ciclo curto), em termos de competição com arroz-vermelho.

Comparações entre espaçamentos entre linhas, para a variável população de plantas, com base na perda unitária (i) e na média dos três cultivares, indicam que o menor espaçamento elevou em 65 e $33 \%$ a competitividade dos cultivares, na primeira e segunda avaliações, respectivamente, em relação ao maior espaçamento (Tabelas 1, 2 e 3). Os cultivares IRGA 417 e BR-IRGA 409 responderam positivamente à redução no espaçamento entre linhas, com aumentos acentuados na competitividade, enquanto BRS-38 Ligeirinho teve o comportamento pouco alterado pela variação desse fator. Nesse sentido, Menezes \& Silva (1998) verificaram, na maioria das combinações de espaçamentos entre linhas e populações de semeadura, que o cultivar de ciclo curto IRGA 416 apresentou menor rendimento de grãos em relação ao cultivar de ciclo médio BR-IRGA 410; essa diferença foi atribuída à menor capacidade competitiva do primeiro cultivar com o arrozvermelho. Já Rieffel Neto et al. (2000) observaram, em situação sem competição interespecífica, que o incremento no espaçamento entre linhas diminuiu o rendimento de grãos de forma diferenciada, dependendo do cultivar.

Ao se compararem os espaçamentos entre linhas para os cultivares que apresentaram maior habilidade competitiva (IRGA 417 e BRIRGA 409), constataram-se aumentos de 125 e $61 \%$ em suas competitividades, respectivamente na primeira e segunda avaliações, quando o espaçamento entre linhas foi reduzido de 25 para $15 \mathrm{~cm}$ (Tabelas 2 e 3). De acordo com Fischer (1985), quando a distância entre fileiras é diminuída, ou quando as plantas estão dispostas de forma eqüidistante, a competição intra-específica é reduzida e favorece-se a capacidade da cultura em competir com as plantas daninhas. Isso se deve ao melhor desenvolvimento individual das plantas cultivadas sob tal condição, comparativamente às plantas dispostas em fileiras mais afastadas.
O modelo não-linear da hipérbole retangular superestimou a assíntota para a maioria das variáveis e condições testadas. Esses resultados podem decorrer do fato de as maiores populações terem sido insuficientes para estimar adequadamente a perda máxima de rendimento. Segundo Cousens (1991), para se obter uma estimativa confiável para o parâmetro $a$, é necessário incluir no experimento populações muito elevadas de plantas daninhas, acima daquelas comumente encontradas sob condições de lavoura.

A utilização das variáveis explicativas cobertura do solo, massa seca da parte aérea e área foliar, como variáveis independentes no modelo de previsão das perdas de rendimento, em geral demonstrou que a área foliar pode ser usada em substituição à variável população de plantas. Nesse sentido, Spitters \& Aerts (1983) afirmam que a associação entre área foliar relativa e perda de rendimento pode ser mais apropriada do que a população de plantas daninhas para predizer a perda de rendimento. De modo semelhante, Vitta \& Fernandez Quintanilla (1996) observaram que o modelo que incorporou a variável área foliar foi tão preciso quanto o da população de plantas daninhas. Cabe ressaltar que a variável área foliar apresentou valores estimados extremamente baixos para perdas unitárias de rendimento de grãos. Além disso, a área foliar não é fácil de medir e sua determinação é demorada, trabalhosa e destrutiva (Vitta \& Fernandez Guintanilla, 1996).

A validação do modelo ajustado, realizada por meio de análise de correlação, apresentou diferenças entre os cultivares testados (Tabela 4). Para o cultivar BRS-38 Ligeirinho não se constatou significância estatística para nenhuma das variáveis e espaçamentos entre linhas testados. Esses resultados podem decorrer da grande variação nos valores obtidos para perdas de rendimento. Atribui-se essa variabilidade à utilização de uma única parcela livre de plantas daninhas (testemunha) e ao ataque do pássaro-preto-dos-arrozais (Gnorimopsar chopi), que podem ter afetado a estimativa do rendimento de grãos do cultivar.

Para o cultivar IRGA 417, a validação do modelo apresentou resultados contraditórios (Tabela 4). A análise de correlação para as variáveis explicativas população de plantas e 
área foliar, no maior espaçamento e na primeira avaliação, não indicou significância estatística. Já para as variáveis massa seca, em ambos os espaçamentos, e área foliar, no menor espaçamento, na primeira avaliação, as correlações foram significativas apenas a $10 \%$ de probabilidade. Para as demais variáveis, em ambas as épocas de avaliação e nos dois espaçamentos, as correlações foram significativas $(\mathrm{p}<0,05)$; nesses casos, os valores de $r$ variaram entre 0,80 e 0,96.

O cultivar BR-IRGA 409 respondeu com significância estatística $(\mathrm{p}<0,05)$ às correlações calculadas para todas as variáveis explicativas e espaçamentos testados, nas duas avaliações

Tabela 4 - Correlações entre perdas do rendimento de grãos de arroz observadas e estimadas em função de população de plantas, cobertura do solo, massa seca da parte área e área foliar do cultivar EEA 406, simulador de arroz-vermelho, IRGA/Cachoeirinha-RS, 2000/01

\begin{tabular}{|c|c|c|c|}
\hline \multirow{2}{*}{$\begin{array}{l}\text { Variável } \\
\text { explicativa }\end{array}$} & \multicolumn{3}{|c|}{ Cultivar de arroz } \\
\hline & $\begin{array}{c}\text { BRS-38 } \\
\text { Ligeirinho }\end{array}$ & IRGA 417 & BR-IRGA 409 \\
\hline \multicolumn{4}{|c|}{$14 \mathrm{DAE}^{\mathrm{i} /}$} \\
\hline $\begin{array}{c}\text { População de plantas } \\
15 \mathrm{~cm}^{2} \\
25 \mathrm{~cm}\end{array}$ & $\begin{array}{c}0,00003^{\mathrm{ns}} \\
0,34^{\mathrm{ns}}\end{array}$ & $\begin{array}{c}0,82^{* *} \\
0,72^{\mathrm{ns}}\end{array}$ & $\begin{array}{l}0,91^{* *} \\
0,96^{* *}\end{array}$ \\
\hline $\begin{array}{c}\text { Cobertura do solo } \\
15 \mathrm{~cm} \\
25 \mathrm{~cm}\end{array}$ & $\begin{array}{c}-0,46^{\mathrm{ns}} \\
0,63^{\mathrm{ns}}\end{array}$ & $\begin{array}{r}0,80 * * \\
0,91 * *\end{array}$ & $\begin{array}{l}0,96 * * \\
0,89 * *\end{array}$ \\
\hline $\begin{array}{l}\text { Massa seca } \\
15 \mathrm{~cm} \\
25 \mathrm{~cm}\end{array}$ & $\begin{array}{l}-0,12^{\mathrm{ns}} \\
0,42^{\mathrm{ns}}\end{array}$ & $\begin{array}{l}0,74^{*} \\
0,86^{*}\end{array}$ & $\begin{array}{l}0,92 * * \\
0,96 * *\end{array}$ \\
\hline $\begin{array}{l}\text { Área foliar } \\
15 \mathrm{~cm} \\
25 \mathrm{~cm}\end{array}$ & $\begin{array}{l}0,13^{\mathrm{ns}} \\
0,42^{\mathrm{ns}}\end{array}$ & $\begin{array}{l}0,72^{*} \\
0,64^{\text {ns }}\end{array}$ & $\begin{array}{l}0,94 * * \\
0,97 * *\end{array}$ \\
\hline \multicolumn{4}{|c|}{28 DAE } \\
\hline $\begin{array}{c}\text { População de plantas } \\
15 \mathrm{~cm} \\
25 \mathrm{~cm}\end{array}$ & $\begin{array}{c}-0,18^{\mathrm{ns}} \\
0,37^{\mathrm{ns}}\end{array}$ & $\begin{array}{r}0,80 * * \\
0,90 * *\end{array}$ & $\begin{array}{l}0,95 * * \\
0,95 * *\end{array}$ \\
\hline $\begin{array}{c}\text { Cobertura do solo } \\
15 \mathrm{~cm} \\
25 \mathrm{~cm}\end{array}$ & $\begin{array}{c}-0,02^{\mathrm{ns}} \\
0,39^{\mathrm{ns}}\end{array}$ & $\begin{array}{l}0,86 * * \\
0,84 * *\end{array}$ & $\begin{array}{l}0,92 * * \\
0,95 * *\end{array}$ \\
\hline $\begin{array}{c}\text { Massa seca } \\
15 \mathrm{~cm} \\
25 \mathrm{~cm}\end{array}$ & $\begin{array}{l}-0,10^{\mathrm{ns}} \\
0,20^{\mathrm{ns}}\end{array}$ & $\begin{array}{l}0,80 * * \\
0,95 * *\end{array}$ & $\begin{array}{l}0,92 * * \\
0,96 * *\end{array}$ \\
\hline $\begin{array}{c}\text { Área foliar } \\
15 \mathrm{~cm} \\
25 \mathrm{~cm}\end{array}$ & $\begin{array}{l}-0,28^{\mathrm{ns}} \\
0,233^{\mathrm{ns}}\end{array}$ & $\begin{array}{l}0,82 * * \\
0,96 * *\end{array}$ & $\begin{array}{l}0,92 * * \\
0,96 * *\end{array}$ \\
\hline
\end{tabular}

${ }^{1 /}$ Dias após a emergência do arroz cultivado;

${ }^{2}$ Espaçamento entre linhas do arroz cultivado. ns - Não-significativo a $5 \%$ de probabilidade. $*$ e ** Significativo a 10 ou $5 \%$ de probabilidade.
(Tabela 4). Os valores para coeficientes de correlação ( $r$ ) indicaram alta associação entre perdas de rendimento de grãos de arroz observadas e estimadas com o uso do modelo, os quais variaram de 0,91 a 0,97.

Em geral, para as variáveis que apresentaram significância estatística, houve melhor associação na segunda avaliação do que na primeira. Esses resultados podem ser devido, em parte, ao ataque do inseto-praga Spodoptera frugiperda, em fase precoce de desenvolvimento da cultura, o que provavelmente alterou os valores iniciais da área foliar. Também, de modo geral, verificaram-se maiores valores de correlação entre perdas de rendimento de grãos de arroz observadas e estimadas para o espaçamento entre linhas de $25 \mathrm{~cm}$, comparativamente ao de $15 \mathrm{~cm}$. Isso pode ser atribuído à maior facilidade na diferenciação entre plantas do cultivar competidor e da cultura e à conseqüente melhor estimativa das variáveis explicativas.

Os resultados obtidos por meio da análise de correlação demonstraram, para os cultivares de arroz de ciclo curto (IRGA 417) e médio (BRIRGA 409), que o modelo proposto por Cousens (1985) pode ser usado na predição da perda de rendimento de arroz irrigado. Desse modo, os resultados que estimam perdas de produtividade podem ser incorporados em modelos, a fim de calcular os níveis de dano econômico para tomada de decisão no controle de arroz-vermelho ou de plantas concorrentes assemelhadas.

Verificou-se que o cultivar IRGA 417 apresenta maior habilidade competitiva com o cultivar EEA 406, simulador de arroz-vermelho, do que os cultivares BRS-38 Ligeirinho e BR-IRGA 409. A redução no espaçamento entre linhas em arroz irrigado aumenta a habilidade competitiva dos cultivares em relação ao cultivar de arroz concorrente, cultivar EEA 406. O modelo de regressão nãolinear da hipérbole retangular estima adequadamente as perdas de rendimento de arroz irrigado ocasionadas pela presença do cultivar EEA 406, simulador de arroz-vermelho. A utilização da variável população de plantas do cultivar concorrente, em modelo de previsão da perda de rendimento de grãos de arroz, em geral permite ajuste satisfatório dos dados para os cultivares estudados. A variável explicativa área foliar do cultivar EEA 406, em 
geral, apresentou equivalência à população de plantas como variável independente no modelo matemático testado.

\section{LITERATURA CITADA}

AGOSTINETTO, D. et al. Arroz vermelho: ecofisiologia e manejo. Ci. Rural, v. 31, n. 2, p. 341-349, 2001.

ARROZ irrigado: recomendações técnicas da pesquisa para o Sul do Brasil. 4.ed. Itajaí: EPAGRI; EMBRAPA, CPACT; IRGA, 1997. 80 p.

ASKEW, S. D.; WILCUT, J. W. Tropic croton interference in cotton. Weed Sci., v. 49, n. 2, p. 184-189, 2001.

BALBINOT Jr., A. A. et al. Competitividade de cultivares de arroz irrigado com cultivar simuladora de arrozvermelho. Pesq. Agropec. Bras., v. 38, n. 1, p. 53-59, 2003.

BERKOWITZ, A. R. Competition for resources in weedcrop mixtures. In: ALTIERI, M. A.; LIEBMAN, M. (Eds.) Weed management in agroecosystems: ecological approaches. Boca Raton: CRC Press, 1988. p. 89-120.

BLACKSHAW, R. E. et al. Integration of cropping practices and herbicides improves weed management in dry bean (Phaseolus vulgaris). Weed Technol., v. 14, n. 2, p. 327-336, 2000.

COUSENS, R. An empirical model relating crop yield to weed and crop density and a statistical comparison with other models. J. Agric. Sci., v. 105, n. 3, p. 513-521, 1985.

COUSENS, R. Aspects of the design and interpretation of competition (interference) experiments. Weed Technol., v. 5 , n. 3 , p. $664-667,1991$.

EMBRAPA CLIMA TEMPERADO. Arroz irrigado: recomendações técnicas da pesquisa para o Sul do Brasil. Pelotas: 1999. 124 p. (Embrapa Clima Temperado. Documento, 57).

FISCHER, A. J. Aspectos de la interferencia entre las malezas y los cultivos. In: SHENK, M.; FISCHER, A. J.; BERNAL, V. (Eds.) Principios básicos sobre el manejo de malezas. El Zamorano: Escuela Agricola Panamericana, 1985. p. 21-40. (Publicación MIPH-EAP, 65).
GRAVOIS, K. A.; HELMS, R. S. Path analysis of rice yield and yield components as affected by seeding rate. Agron. J., v. 84, n. 1, p. 1-4, 1992.

MENEZES, V. G.; SILVA, P. R. F. Manejo de arroz vermelho através do tipo e arranjo de plantas em arroz irrigado. Planta Daninha, v. 16, n. 1, p. 45-58, 1998.

NGOUAJIO, M.; LEMIEUX, C.; LEROUX, G. D. Prediction of corn (Zea mays) yield loss from early observations of the relative leaf area and the relative leaf cover of weeds. Weed Sci., v. 47, n. 3, p. 297-304, 1999.

NI, H. et al. Oryza sativa plant traits conferring competitive ability against weeds. Weed Sci., v. 48, n. 2, p. 200-204, 2000.

RATKOWSKY, D. A. Nonlinear regression modeling: a unified practical approach. New York: Marcel Dekker, 1983. p. $135-154$.

RIEFFEL NETO, S. R. et al. Resposta de genótipos de arroz irrigado ao arranjo de plantas. Pesq. Agropec. Bras., v. 35 , n. 12 , p. $2383-2390,2000$.

SAS - Institute Statistical Analysis System. User's guide: version 6. 4.ed. Cary: 1989. 846 p.

SPITTERS, C. J. T.; AERTS, R. Simulation of competition for light and water in crop-weed associations. Aspects Appl. Biol., v. 4, n. 2, p. 467-483, 1983.

SWINTON, S. M. et al. Estimation of crop yield loss due to interference by multiple weed species. Weed Sc., Champaign, v. 42, n. 1, p. 103-109, 1994.

SWINTON, S. M.; LYFORD, C. P. A test for choice between hyperbolic and sigmoidal models of crop yield response to weed density. J. Agric. Biol. Environ. Stat., v. 1, n. 1, p. 97-106, 1996.

VITTA, J. I.; FERNANDEZ QUINTANILLA, C. Canopy measurements as predictors of weed-crop competition. Weed Sci., v. 44, n. 3, p. 511-516, 1996.

YENISH, J. P. et al. Wheat (Triticum aestivum) yield reduction from common milkweed (Asclepias syriaca) competition. Weed Sci., v. 45, n. 1, p. 127-131, 1997. 